\title{
MAGNETIC RESONANCE BRAIN IMAGE SEGMENTATION
}

\author{
M.C.Jobin Christ ${ }^{1}$ and Dr.R.M.S.Parvathi ${ }^{2}$ \\ ${ }^{1}$ Adhiyamaan College of Engineering, Dr.MGR Nagar, Hosur, India \\ jobinchrist@gmail. com \\ ${ }^{2}$ Sengunthar College of Engineering, Tiruchengode, India \\ rmsparvathi@india.com
}

\begin{abstract}
Segmentation of tissues and structures from medical images is the first step in many image analysis applications developed for medical diagnosis. With the growing research on medical image segmentation, it is essential to categorize the research outcomes and provide researchers with an overview of the existing segmentation techniques in medical images. In this paper, different image segmentation methods applied on magnetic resonance brain images are reviewed. The selection of methods includes sources from image processing journals, conferences, books, dissertations and thesis. The conceptual details of the methods are explained and mathematical details are avoided for simplicity. Both broad and detailed categorizations of reviewed segmentation techniques are provided. The state of art research is provided with emphasis on developed techniques and image properties used by them. The methods defined are not always mutually independent. Hence, their inter relationships are also stated. Finally, conclusions are drawn summarizing commonly used techniques and their complexities in application.
\end{abstract}

\section{KEYWORDS}

MRI, Segmentation, Medical Imaging

\section{INTRODUCTION}

Image processing consists of various application fields like compression, enhancement, detection, feature extraction, restoration, scaling, segmentation, etc. Image segmentation is used in various applications like medical imaging, locating objects in satellite images, face recognition, traffic control systems, fingerprint recognition and machine vision etc. Medical imaging includes locating tumors and other pathologies, measuring tissue volumes, etc. Segmentation plays an important role in biomedical image processing. Segmentation is the starting point for other processes such as registration, shape analysis, visualization and quantitative analysis. Segmentation of an image is the division or separation of the image into disjoint regions of similar attribute. In clinical practice, Magnetic Resonance Imaging is used to distinguish pathologic tissue from normal tissue, especially for brain related disorders. Three main regions of brain, White Matter (WM), Gray Matter (GM) and Cerebrospinal Fluid (CSF) are the important subject of study in brain imaging. Manual segmentation by an expert is time consuming and it is 
International Journal of VLSI design \& Communication Systems (VLSICS) Vol.3, No.4, August 2012

very difficult to do accurate segmentation. Hence automatic segmentation algorithms are preferred in diagnostic process.

\section{IMAGE SEGMENTATION METHODS}

Image segmentation algorithms are classified into two types, supervised and unsupervised. Unsupervised algorithms are fully automatic and partition the regions in feature space with high density. The different unsupervised algorithms are Feature-Space based Techniques, Clustering (K-means algorithm, C-means algorithm, E-means algorithm), Histogram thresholding, ImageDomain or Region Based Techniques (Split-and-merge techniques, Region growing techniques, Neural-network based techniques, Edge Detection Technique), Fuzzy Techniques, Hybrid techniques, etc. It is essential to know which method is to be applicable for the segmentation of medical images. In this paper we present a comparative study of unsupervised algorithms in terms of robustness, accuracy $[3,7,10]$.

\subsection{Clustering Methods}

Clustering is a method of grouping a set of patterns into a number of clusters such that similar patterns are assigned to one cluster. Each pattern can be represented by a vector having many attributes. Clustering technique is based on the computation of a measure of similarity or distance between the respective patterns. In this paper we are going to discuss about K-means algorithm, Fuzzy C-means algorithm.

\subsubsection{K-means algorithm}

K-means algorithm is under the category of Squared Error-Based Clustering (Vector Quantization) and it is also under the category of crisp clustering or hard clustering. K-means algorithm is very simple and can be easily implemented in solving many practical problems. Steps of the K-means algorithm are given below.

1. Choose $\mathrm{k}$ cluster centers to coincide with $\mathrm{k}$ randomly chosen patterns inside the hyper volume containing the pattern set.(C)

2. Assign each pattern to the closest cluster center. $\left(\mathrm{C}_{\mathrm{i}, \mathrm{i}} \mathrm{i}=1,2, \ldots \mathrm{C}\right)$

3. Recompute the cluster centers using the current cluster memberships.(U)

4. If a convergence criterion is not met, go to step 2 with new cluster centers by the following equation, i.e., minimal decrease in squared error.

$$
\left\{\begin{array}{l}
1 \text { if }\left\|x_{j}-\left.C_{i}\right|^{2} \leq\right\| x_{j}-C_{k} \|^{2}, \text { for each } k \neq i \\
\text { o otherwise }
\end{array}\right.
$$

Where, $\left|G_{i}\right|$ is the size of $G_{i}$

or, 
International Journal of VLSI design \& Communication Systems (VLSICS) Vol.3, No.4, August 2012

$$
\left|G_{i}\right|=\sum_{j=1}^{n} U_{i j}
$$

The performance of the K-means algorithm depends on the initial positions of the cluster centers. This is an inherently iterative algorithm. And also there is no guarantee about the convergence towards an optimum solution. The convergence centroids vary with different initial points. It is also sensitive to noise and outliers. It is only based on numerical variables [1, 4, 8, 12 ].

\subsubsection{Fuzzy C-Means algorithm}

Fuzzy C-Means clustering (FCM), also called as ISODATA, is a data clustering method in which each data point belongs to a cluster to a degree specified by a membership value. FCM is used in many applications like pattern recognition, classification, image segmentation, etc. FCM divides a collection of $n$ vectors $c$ fuzzy groups, and finds a cluster center in each group such that a cost function of dissimilarity measure is minimized. FCM uses fuzzy partitioning such that a given data point can belong to several groups with the degree of belongingness specified by membership values between 0 and 1. This algorithm is simply an iterated procedure. The algorithm is given below.

1) Initialize the membership matrix $\mathbf{U}$ with random values between 0 and 1.

2) Calculates c fuzzy cluster center $\mathrm{c}_{\mathrm{i}}, i=1, \ldots c$, using the following equation,

$$
c_{i}=\frac{\sum_{j=1}^{n} u_{i j}^{m} x_{j}}{\sum_{j=1}^{n} u_{i j}^{m}}
$$

3) Compute the cost by the following equation. Stop if either it is below a certain threshold value or its improvement over previous iteration.

$$
J\left(U, c_{1}, \ldots, c_{c}\right)=\sum_{i=1}^{c} J_{i}=\sum_{i=1}^{c} \sum_{j}^{n} u_{i j}^{m} d_{i j}^{2}
$$

4) Compute a new $\mathbf{U}$ by the equation. Go to step 2.

$$
u_{i j}=\frac{1}{\sum_{k=1}^{c}\left(\frac{d_{i j}}{d_{k j}}\right)^{2 /(m-1)}}
$$

Like K-means clustering, there is no guarantee ensures that FCM converges to an optimum solution. The performance is based on the initial cluster centers. FCM also suffers from the presence of outliers and noise and it is difficult to identify the initial partitions [1, 8, 12 ].

\subsection{Edge detection methods}

Edge detection is a method which is extensively used for gray level image segmentation. It is a process of finding the discontinuities of an image. Edge detection is under the category of Boundary based technique. Boundary based methods find connected regions based on finding pixel differences of the pixels within them. The objective is to find a closed boundary such that an outside can be determined easily. Edge Detection process is classified into two broad groups; (i) 
International Journal of VLSI design \& Communication Systems (VLSICS) Vol.3, No.4, August 2012

Derivative approach, (ii) Pattern fitting approach. Both approaches have advantages as well as disadvantages. And also second method gives better result as compared with the first method. Pattern fitting approach uses a series of edge approximation functions over a small neighborhood and it will be analyzed. In derivative approach, edge pixels are found by taking derivatives. Here edge masks are used to find two dimensional derivatives. In this chapter we will discuss about the Roberts, Prewitt, Sobel, Canny, Gaussian, LoG operators in detail. They are under the category of Derivative approach $[3,4,10]$.

\subsubsection{Roberts Operator}

This method is based on differences between adjacent pixels. Here, +1 and -1 are explicitly used to find the edges. This difference is called as forward differences. The first order partial derivative is implemented by cross-gradient operator.

$$
\begin{aligned}
& d_{1}=g_{0}-g_{2}(7) \\
& d_{2}=g_{1}-g_{3}(8)
\end{aligned}
$$

The above two partial derivatives are implemented by approximating them into $2 \times 2$ windows. The Roberts masks are,

\begin{tabular}{|l|l|}
\hline-1 & 0 \\
\hline 0 & 1 \\
\hline
\end{tabular}

\begin{tabular}{|l|l|}
\hline 0 & 1 \\
\hline-1 & 0 \\
\hline
\end{tabular}

Figure 1. Roberts windows

\subsubsection{Prewitt Operator}

In Prewitt operator, similar weights are assigned to all the neighbours of the chosen pixel. The first order derivative is given by,

$$
\begin{aligned}
& d_{1}=1 / 3\left[\left(g_{4}+g_{5}+g_{6}\right)-\left(g_{2}+g_{1}+g_{8}\right)\right] \\
& d_{2}=1 / 3\left[\left(g_{8}+g_{7}+g_{6}\right)-\left(g_{2}+g_{3}+g_{4}\right)\right]
\end{aligned}
$$

Its masks are given below,

\begin{tabular}{|c|c|c|}
\hline-1 & -2 & -1 \\
\hline 0 & 0 & 0 \\
\hline 1 & 2 & 1 \\
\hline
\end{tabular}

\begin{tabular}{|l|l|l|}
\hline-1 & 0 & 1 \\
\hline-2 & 0 & 2 \\
\hline-1 & 0 & 1 \\
\hline
\end{tabular}

Figure 2. Prewitt windows

\subsubsection{Sobel Operator}

In Sobel operator, higher weights are assigned to the close neighbours of the chosen pixel. The first order derivative is given by,

$$
\begin{aligned}
& d_{1}=1 / 4\left[\left(g_{4}+2 g_{5}+g_{6}\right)-\left(g_{2}+2 g_{1}+g_{8}\right)\right] \\
& d_{2}=1 / 4\left[\left(g_{8}+2 g_{7}+g_{6}\right)-\left(g_{2}+2 g_{3}+g_{4}\right)\right]
\end{aligned}
$$


International Journal of VLSI design \& Communication Systems (VLSICS) Vol.3, No.4, August 2012

Sobel Masks are given below,

\begin{tabular}{|l|l|l|}
\hline-1 & 0 & 1 \\
\hline-1 & 0 & 1 \\
\hline-1 & 0 & 1 \\
\hline
\end{tabular}

\begin{tabular}{|c|c|c|}
\hline-1 & -1 & -1 \\
\hline 0 & 0 & 0 \\
\hline 1 & 1 & 1 \\
\hline
\end{tabular}

Figure 3. Sobel windows

\subsubsection{Canny Operator}

It is the most popular operator among all the edge detection algorithms. Canny algorithm mainly concentrate on three things, Maximizing Signal to Noise Ratio (SNR), localization of edges by minimizing the variance of the zero crossing position, identification of single edge rather than multiple response. The canny algorithm is given below,

i. Apply derivative of Gaussian

ii. Non-maximum suppression

iii. $\quad$ Linking and thresholding

\subsubsection{LoG Operator}

Laplacian of Gaussian operator is otherwise called as Marr-Hildreth operator. It is based on the second derivative method for the detection of zero crossing method. Here in addition to the Laplacian operator, Gaussian smoothing is applied. Laplacian mask is given below,

\begin{tabular}{|c|c|c|}
\hline 0 & 1 & 0 \\
\hline 1 & -4 & 1 \\
\hline 0 & 1 & 0 \\
\hline
\end{tabular}

Figure 4. Laplacian Window

LoG algorithm is given below.

i. Smooth the image by convolving it with a digital mask.

ii. Apply the Laplacian mask.

iii. Find the zero crossings by Laplacian second derivative operator.

\subsection{Watershed method}

Watersheds are one of the typical regions in the field of topography. A drop of the water falling it flows down until it reaches the bottom of the region. Monochrome image is considered to be an height surface in which high-altitude pixels correspond to ridges and low-altitude pixels correspond to valleys. This suggestion says if we have a minima point, by falling water, region and the frontier can be achieved. Watershed uses image gradient to initial point and region can get by region growing. The accretion of water in the neighborhood of local minima is called a catchment basin. Watershed refers to a ridge that divides areas shattered by different river systems. A catchment basin is the environmental area draining into a river or reservoir. If we consider that bright areas are high and dark areas are low, then it might look like the plane. With planes, it is natural to think in terms of catchment basins and watershed lines. Two approaches are there to find watershed of an image, 
1. Rainfall approach

2. Flooding approach

In rainfall approach, local minima are found all through the image, and each local minima is assigned an exclusive tag. A intangible water drop is placed at each untagged pixel. The drop moves to low amplitude neighbor until it reaches a tagged pixel and it assumes tag value. In flooding approach, intangible pixel holes are pierced at each local minima. The water enters the holes and takings to fill each catchment basin. If the basin is about to overflow, a dam is built on its neighboring ridge line to the height of high altitude ridge point. These dam borders correspond to the watershed lines. The following steps are used in Watershed Algorithm:

i. $\quad$ Read an Image and covert it into grayscale

ii. Use gradient magnitude as the segmentation function

iii. Mark the foreground objects

iv. Calculate the Background markers

v. Calculate the watershed transform of the segmentation function

vi. Visualize the result

The main drawback of this algorithm is over segmentation, because all of edge and noise would appear in the image gradient. If the signal to noise ratio is not high enough at the contour of interest, the transform won't detect it correctly. It also failed to detect thin structures $[4,7,10]$.

\subsection{Region based methods}

Regions are group of connected pixel elements with similar properties. In this method each pixel element is assigned to a particular region. Region growing is a process that groups pixels or sub regions into larger regions. In which nearest pixel elements are examined and added to a region if no edges are detected. It starts with a set of "seed" points and from these produces regions by adding to each seed points those nearest pixels that have similar properties. Region splitting is another region based approach. It starts with a entire image and divides it into homogeneous regions. Splitting method alone not sufficient for segmentation process. Therefore merging will be applied after splitting, which is called as split and merge method. Steps of Split and merge algorithm is given below.

1. If the entire region is consistent, leave it unchanged.

2. If the region is not sufficiently consistent, split it into four quadrants.

3. Merge any adjacent regions that are similar enough.

4. Repeat steps 2 and 3 repeatedly until no more splitting or merging arises $[9,10]$.

\subsection{Thresholding methods}

Thresholding methods give segments having picture elements with similar gray levels. This technique requires that an object has homogenous gray level and a background with a different gray level. That kind of image can be segmented by two regions using thresholding. Thresholding techniques are classified into Global or fixed thresholding, adaptive thresholding and histogram based thresholding. In this chapter we described OTSU method which is under the category of histogram based thresholding. This method is simple and is an outstanding method for selecting the threshold. For a gray scale image, the total number of pixels is defined as $N, n_{i}$ is the number of pixels which's intensity is $i$. By regularizing the histogram, the following equations could be attained. 
International Journal of VLSI design \& Communication Systems (VLSICS) Vol.3, No.4, August 2012

$$
\begin{gathered}
\sum_{i=0}^{255} n_{i}=N \\
p_{i}=\frac{n_{i}}{N}
\end{gathered}
$$

$p_{i}$ is the probability of the pixels which's intensity is $i$. The threshold of the image segmentation is defined as $m$, then the probability $\theta_{\mathrm{o}}$ and mean value $\mu_{\mathrm{o}}$ of the background can be attained through the following equations:

$$
\begin{array}{r}
\theta_{\mathrm{o}}=\sum_{i=\mathrm{o}}^{m} p_{i} \\
\mu_{\mathrm{o}}=\frac{\sum_{i=\mathrm{o}}^{m} i p_{i}}{\theta_{\mathrm{o}}}
\end{array}
$$

probability and typical value of the target also can be obtained:

$$
\begin{aligned}
& \theta_{1}=\sum_{i=m+1}^{255} i p_{i} \\
& \mu_{1}=\frac{\sum_{t=m+1}^{255} i p_{i}}{\theta_{1}}
\end{aligned}
$$

By computing all the above values, the following equation is attained,

$$
\sigma_{B}^{2}=\theta_{0} \theta_{1}\left(\mu_{0}-\mu_{1}\right)^{2}
$$

The threshold which makes the variance yields maximal is the optimal threshold $[4,7]$.

\subsection{Neural Network based Methods}

Artificial Neural Networks (ANNs) have been developed for range of applications such as function approximation, feature extraction, optimization, pattern recognition and classification. Particularly, they have been developed for image enhancement, segmentation, registration, feature extraction, and object recognition. Among the above applications, image segmentation is more important as it is a crucial step for high level processing such as detection of tumors in medical images. Multi-Layer Perceptron (MLP), Radial Basis Function (RBF), Hopfield, Back Propagation Networks (BPN), Self Organized Map (SOM), Adaptive Resonance Theory (ART), Cellular, and Pulse-Coupled neural networks have been used for image segmentation. These networks can be categorized into feed forward (associative) and feedback (auto associative) networks. MLP, Self Organized Map (SOM), and RBF neural networks belong to the feedforward networks [6]. In this paper we describe about Self Organized Map.

A SOM is a single layer feed forward neural network. SOMs are generally referred as Kohonen's Self Organizing Map. They are also called as Kohonen Neural Networks, Topological Neural Networks or Self-organizing Feature Maps (SOFM). It was discovered by Kohonen in 1982. 
International Journal of VLSI design \& Communication Systems (VLSICS) Vol.3, No.4, August 2012

Kohonen described SOM as a "visualization and analysis tool for high dimensional data." These are really the two most attractive characteristics of SOM. SOM can be used as an alternative of conventional K-means clustering algorithm. But SOM is having more advantages than the Kmeans clustering algorithm. The main advantage of SOM is that it is minimum chance to local minima than the conventional K-means clustering algorithm, and it can be acted as a good initialization algorithm for that method. The main advantage is clusters obtained using SOM are topologically ordered. SOM projects multidimensional data onto one or two dimensions. So we can easily visualize the data in these dimensions. SOM is also used as a supervised classification by labeling the neurons (or units) with the classes of the data that are mapped to it [13]. Training Algorithm of SOM is given below.

Let $\quad x_{k}(k=1$ to the number of training patterns $N)$ be the $n$ dimensional training patterns.

$w_{i j}$ be the unit in position $(i, j)$.

$0 \leq \alpha \leq 1$ be the learning rate.

$h\left(w_{i j}, w_{m n}, r\right)$ be the neighborhood function. The neighborhood function has values in the range $[0,1]$. It is high for units that are close in the output space and small for units far away. Usually select a function that is 1 if $w_{i j}=w_{m n}$, monotonically decreases as the distance in the grid between them increases up to a radius $r$, neighborhood radius.

$w_{b m u}$ be the best matching unit for a given input pattern.

The training algorithm consists of the following steps,

For each input pattern $x_{k,}$

1. Calculate the distances between the pattern $x_{k}$ and all units $w_{i j}$

$$
d_{i j}=\left\|x_{k}-w_{i j}\right\|
$$

2. Select the nearest unit $w_{i j}$ as best matching unit $w_{b m u}=w_{i j}$

$$
d_{i j}=\min \left(d_{m n}\right)
$$

3. Update each unit $w_{i j}$ according to the rule

$$
w_{i j}=w_{i j}+\alpha h\left(w_{b m u}, w_{i j}, r\right)\left\|x_{k}-w_{i j}\right\|
$$

4. Repeat the process until convergence is reached.

\subsection{Hybrid methods}

Every method is having its own merits as well as demerits depend upon their applications. So in order to strengthen the methods, hybridization integrates the technologies by suppressing their weakness. We may integrate fuzzy logic with neural networks, fuzzy logic with genetic algorithms, genetic algorithm with neural networks and so [11]. In medical image segmentation, instead of applying a single technology if we are applying multiple technologies together means it will produce efficient results. In this section we present hybridization between K-Means (KM) algorithm with Marker controlled watershed algorithm and Fuzzy C-Means algorithm with Marker controlled watershed algorithm. This is a two stage process. The first process uses Kmeans clustering to produce a primary segmentation of the input image, while the second process applies the marker controlled watershed segmentation algorithm to the primary segmentation to 
International Journal of VLSI design \& Communication Systems (VLSICS) Vol.3, No.4, August 2012

obtain the final segmentation map. Similarly we can modify the above process by Fuzzy C-Means clustering instead of K-means clustering [5].

\subsection{Other methods}

In addition to the above mentioned algorithms, Texture based methods, Wavelet based methods, Level set methods, Wavelength based method, Genetic algorithm based method, etc. also used for medical image segmentation. Each method is having its own advantages as well as limitations [2].

\section{EXECUTION}

The above methods are implemented using MATLAB 7.9.0(R2009b). MRI brain image is taken for implementation. JPEG image file format is used.

\section{RESULTS}

Results of various algorithms are given below.

\subsection{Results of Clustering Methods}

\begin{tabular}{|c|c|}
\hline Input Image \\
\hline $\begin{array}{c}\text { FCM with 4 } \\
\text { cluster centres }\end{array}$ \\
\hline $\begin{array}{c}\text { FCM with 3 } \\
\text { cluster centres }\end{array}$ \\
\hline $\begin{array}{c}\text { KM with } 4 \\
\text { cluster centres }\end{array}$ \\
\hline $\begin{array}{c}\text { KM with } 3 \\
\text { cluster centres }\end{array}$ \\
\hline
\end{tabular}

Figure 5. Clustering Methods

\subsection{Results of Edge detection methods}

\begin{tabular}{|c|c|}
\hline Input Image & \\
\hline Roberts \\
Operator \\
\hline Prewitt \\
Operator \\
\hline
\end{tabular}


International Journal of VLSI design \& Communication Systems (VLSICS) Vol.3, No.4, August 2012

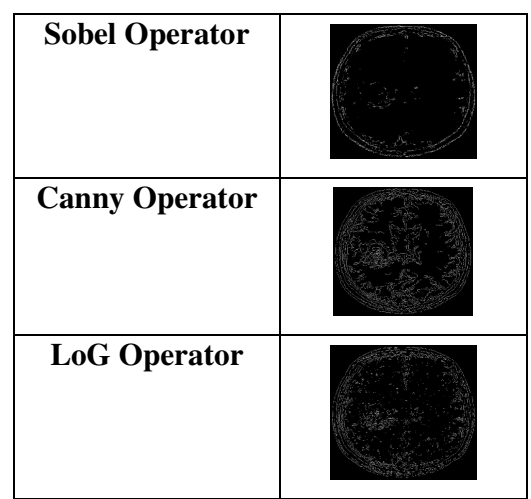

Figure 6. Edge detection methods

\subsection{Results of Watershed method}

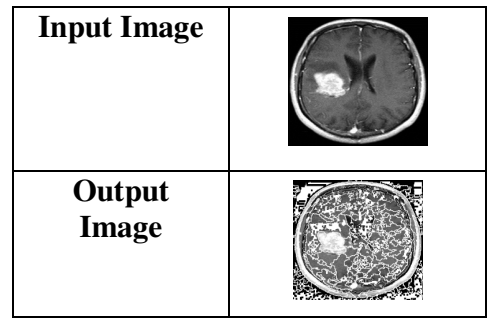

Figure 7. Watershed method

\subsection{Results of Split and merge method}

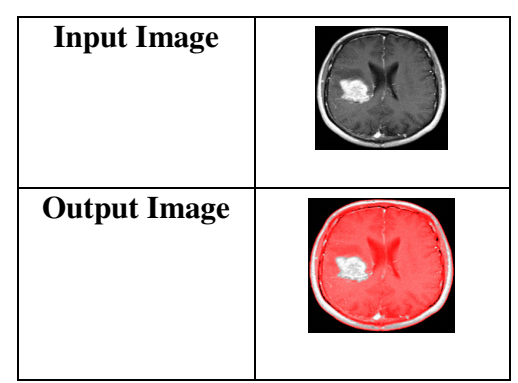

Figure 8. Split and merge method

\subsection{Results of Thresholding method}

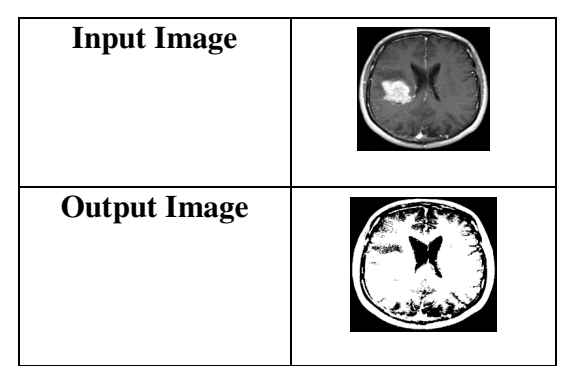

Figure 9. Thresholding method 


\subsection{Results of SOM based method}

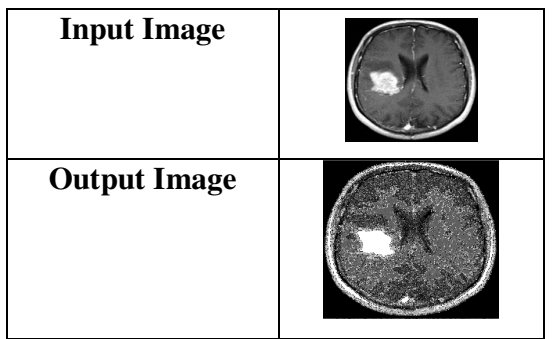

Figure 10. SOM based method

\subsection{Results of K-Means clustering with Marker controlled watershed method}

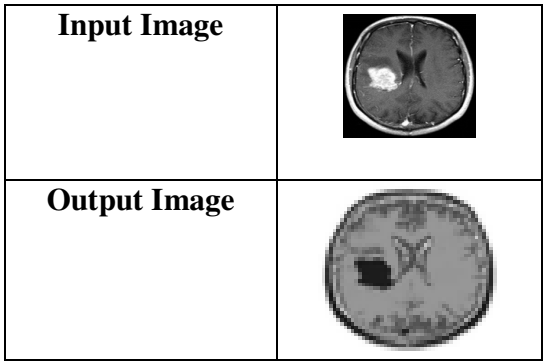

Figure 11. K -Means clustering with Marker controlled watershed method

\subsection{Results of Fuzzy C-Means clustering with Marker controlled watershed method}

\begin{tabular}{|c|c|}
\hline Input Image & \\
\hline Output Image & \\
\hline
\end{tabular}

Figure 12. Fuzzy C-Means clustering with Marker controlled watershed method

\section{CONCLUSIONS}

The different segmentation methods have been demonstrated. From the results, the clustering algorithms are guaranteed to converge but it may not return optimal solution. In K-Means algorithm the quality of the solution depends on the initial set of clusters and value of K. An inapt choice of $\mathrm{K}$ yields very poor result. But for White matter segmentation, it gave better results. From the results of edge detection techniques, the canny operator performed well than all other operators. In watershed algorithms, the length of gradients is taken as elevation information. The flooding process is performed over gradient image, this leads to an over segmentation of an image. Split and Merge technique operated well over all images. Mainly it is suitable for detection of tumors, etc. Thresholding technique is not suited for WM, GM and CSF Segmentation. But it is giving satisfactory results for tumor affected images. SOM is more effective in the case of tumor segmentation. And it can be used as a perfect substitute of traditional clustering algorithms. Hybrid methods of using K-means clustering and Fuzzy C 
International Journal of VLSI design \& Communication Systems (VLSICS) Vol.3, No.4, August 2012

Means clustering exclusively to obtain a primary segmentation of MR brain images before applying the marker controlled watershed segmentation to them is effective. And integration of K-means clustering with marker controlled watershed algorithm gave better segmentation than integration of Fuzzy C-means clustering with marker controlled watershed algorithm.

\section{REFERENCES}

[1] A.K.Jain, et.al (1999), Data Clustering: A Review, ACM Computing Surveys, Vol.31, No. 3

[2] B. B. Chaudhuri and Nirupam Sarkar (1995), Texture Segmentation Using Fractal Dimension, IEEE transactions on pattern analysis and machine intelligence, vol. 17, no. 1

[3] B.Chanda, D.Dutta Majumder (2008), Digital Image Processing and Analysis, Prentice Hall of India Pvt. Ltd

[4] H.S.Prasantha et. Al (2010), Medical Image Segmentation, International Journal on Computer Science and Engineering, Vol. 02, No. 04

[5] M.C.Jobin Christ and R.M.S.Parvathi (2011), Segmentation of Medical Image using Clustering and Watershed Algorithms, American Journal of Applied Sciences, Vol. 8, No. 12, pp: 1349-1352

[6] Mostafa Jabarouti Moghaddam and Hamid Soltanian-Zadeh, Medical Image Segmentation Using Artificial Neural Networks, Artificial Neural Networks - Methodological Advances and Biomedical Applications, pp. 121-138

[7] Rafael C. Gonzalez, Richard E. Woods (2007), Digital Image processing, 2nd edition, Pearson Education, pp.589-656

[8] Rui Xu and Donald Wunsch II (2005), Survey of clustering algorithm, IEEE transactions on neural networks, Vol. 16, No. 3

[9] S. A. Hojjatoleslami and J. Kittler (1998), Region Growing: A New Approach, IEEE Transactions on Image Processing, Vol. 7, No. 7

[10] S.Jayaraman, et.al (2009), Digital Image Processing, Tata McGraw Hill Education Pvt. Ltd

[11] S.Rajasekaran and G.A. Vijayalakshmi Pai (2011), Neural Networks, Fuzzy Logic, and Genetic Algorithms Synthesis and Applications, PHI Learning Private Ltd., pp : 297-300

[12] Veronica S. Moertini (2002), Introduction to five data clustering algorithms, INTEGRAL, Vol. 7, No. 2

[13] Victor .J.A.S. Lobo (2009), Application of Self-Organizing Maps to the Maritime Environment, Information Fusion and Geographic Information Systems, Lecture Notes in Geo information and Cartography, DOI: 10.1007/978-3-642-00304-2_2, ( S Springer-Verlag Berlin Heidelberg 
International Journal of VLSI design \& Communication Systems (VLSICS) Vol.3, No.4, August 2012

\section{Authors}

M.C.Jobin Christ received Bachelor degree from Manonmaniam Sundaranar University, Tirunelveli and Master Degree from Anna University, Chennai. Currently he is pursuing $\mathrm{PhD}$ in Anna University, Coimbatore in the area of Image Processing. His research interest includes Digital Image Processing, Digital Signal Processing and Artificial Neural Networks. Currently he is working as an Assistant Professor in the department of Biomedical Engineering at Adhiyamaan College of Engineering, Hosur. He is having total teaching experience of 7 years. He is a life member of BMESI.

Dr. R.M.S Parvathi has studied B.E. (ECE) and M.E.(CSE) from Govt. College of Technology, Coimbatore, and obtained Ph.D. in Computer Science \& Engineering from Bharathiar University Coimbatore. Currently she is working as a principal in Sengunthar College of Engineering, Tiruchengode. Dr. R.M.S. Parvathi is having total teaching experience of 22 years both in UG \& PG Engineering. Also having 11 year Industrial, R \& D Experience. Dr. R.M.S. Parvathi has published nearly 56 technical papers in both national and International conference. Dr.R.M.S. Parvathi has also published 64

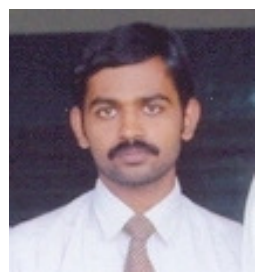
technical papers in National \& International journals. Dr. R.M.S. Parvathi is life member of ISTE and having memberships in CSI and she is a fellow member of IEEE. Dr. R.M.S. Parvathi is presently guiding students for doctoral program. Also Dr. R.M.S. Parvathi is authoring 2 books on UML. 